\title{
The Second Dialog State Tracking Challenge
}

\author{
Matthew Henderson $^{1}$, Blaise Thomson ${ }^{1}$ and Jason Williams ${ }^{2}$ \\ ${ }^{1}$ Department of Engineering, University of Cambridge, U.K. \\ ${ }^{2}$ Microsoft Research, Redmond, WA, USA \\ mh521@eng.cam.ac.uk brmt2@eng.cam.ac.uk jason.williams@microsoft.com
}

\begin{abstract}
A spoken dialog system, while communicating with a user, must keep track of what the user wants from the system at each step. This process, termed dialog state tracking, is essential for a successful dialog system as it directly informs the system's actions. The first Dialog State Tracking Challenge allowed for evaluation of different dialog state tracking techniques, providing common testbeds and evaluation suites. This paper presents a second challenge, which continues this tradition and introduces some additional features - a new domain, changing user goals and a richer dialog state. The challenge received 31 entries from 9 research groups. The results suggest that while large improvements on a competitive baseline are possible, trackers are still prone to degradation in mismatched conditions. An investigation into ensemble learning demonstrates the most accurate tracking can be achieved by combining multiple trackers.
\end{abstract}

\section{Introduction}

Spoken language provides a medium of communication that is natural to users as well as hands- and eyes-free. Voice-based computer systems, called spoken dialog systems, allow users to interact using speech to achieve a goal. Efficient operation of a spoken dialog system requires a component that can track what has happened in a dialog, incorporating system outputs, user speech and context from previous turns. The building and evaluation of these trackers is an important field of research since the performance of dialog state tracking is important for the final performance of a complete system.
Until recently, it was difficult to compare approaches to state tracking because of the wide variety of metrics and corpora used for evaluation. The first dialog state tracking challenge (DSTC1) attempted to overcome this by defining a challenge task with standard test conditions, freely available corpora and open access (Williams et al., 2013). This paper presents the results of a second challenge, which continues in this tradition with the inclusion of additional features relevant to the research community.

Some key differences to the first challenge include:

- The domain is restaurant search instead of bus timetable information. This provides participants with a different category of interaction where there is a database of matching entities.

- Users' goals are permitted to change. In the first challenge, the user was assumed to always want a specific bus journey. In this challenge the user's goal can change. For example, they may want a 'Chinese' restaurant at the start of the dialog but change to wanting 'Italian' food by the end.

- The dialog state uses a richer representation than in DSTC1, including not only the slot/value attributes of the user goal, but also their search method, and what information they wanted the system to read out.

As well as presenting the results of the different state trackers, this paper attempts to obtain some insights into research progress by analysing their performance. This includes analyses of the predictive power of performance on the development set, the effects of tracking the dialog state using joint distributions, and the correlation between 1-best accuracy and overall quality of probability distributions output by trackers. An evaluation of the effects of ensemble learning is also performed.

The paper begins with an overview of the chal- 
lenge in section 2. The labelling scheme and metrics used for evaluation are discussed in section 3 followed by a summary of the results of the challenge in section 4. An analysis of ensemble learning is presented in section 5. Section 6 concludes the paper.

\section{Challenge overview}

\subsection{Problem statement}

This section defines the problem of dialog state tracking as it is presented in the challenge. The challenge evaluates state tracking for dialogs where users search for restaurants by specifying constraints, and may ask for information such as the phone number. The dialog state is formulated in a manner which is general to information browsing tasks such as this.

Included with the data is an ontology ${ }^{1}$, which gives details of all possible dialog states. The ontology includes a list of attributes termed requestable slots which the user may request, such as the food type or phone number. It also provides a list of informable slots which are attributes that may be provided as constraints. Each informable slot has a set of possible values. Table 1 gives details on the ontology used in DSTC2.

The dialog state at each turn consists of three components:

- The goal constraint for each informable slot. This is either an assignment of a value from the ontology which the user has specified as a constraint, or is a special value - either Dontcare which means the user has no preference, or None which means the user is yet to specify a valid goal for this slot.

- A set of requested slots, i.e. those slots whose values have been requested by the user, and should be informed by the system.

- An assignment of the current dialog search method. This is one of

- by constraints, if the user is attempting to issue a constraint,

- by alternatives, if the user is requesting alternative suitable venues,

- by name, if the user is attempting to ask about a specific venue by its name,

- finished, if the user wants to end the call

- or none otherwise.

Note that in DSTC1, the set of dialog states

\footnotetext{
${ }^{1}$ Note that this ontology includes only the schema for dialog states and not the database entries
}

was dependent on the hypotheses given by a Spoken Language Understanding component (SLU) (Williams et al., 2013), whereas here the state is labelled independently of any SLU (see section 3). Appendix B gives an example dialog with the state labelled at each turn.

A tracker must use information up to a given turn in the dialog, and output a probability distribution over dialog states for the turn. Trackers output separately the distributions for goal constraints, requested slots and the method. They may either report a joint distribution over the goal constraints, or supply marginal distributions and let the joint goal constraint distribution be calculated as a product of the marginals.

\subsection{Challenge design}

DSTC2 studies the problem of dialog state tracking as a corpus-based task, similar to DSTC1. The challenge task is to re-run dialog state tracking over a test corpus of dialogs.

A corpus-based challenge means all trackers are evaluated on the same dialogs, allowing direct comparison between trackers. There is also no need for teams to expend time and money in building an end-to-end system and getting users, meaning a low barrier to entry.

When a tracker is deployed, it will inevitably alter the performance of the dialog system it is part of, relative to any previously collected dialogs. In order to simulate this, and to penalise overfitting to known conditions, evaluation dialogs in the challenge are drawn from dialogs with a dialog manager which is not found in the training data.

\subsection{Data}

A large corpus of dialogs with various telephonebased dialog systems was collected using Amazon Mechanical Turk. The dialogs used in the challenge come from 6 conditions; all combinations of 3 dialog managers and 2 speech recognisers. There are roughly 500 dialogs in each condition, of average length 7.88 turns from 184 unique callers.

The 3 dialog managers are:

- DM-HC, a simple tracker maintaining a single top dialog state, and a hand-crafted policy

- DM-POMDPHC, a dynamic Bayesian network for tracking a distribution of dialog states, and a hand-crafted policy

- DM-POMDP, the same tracking method as DM-POMDPHC, with a policy learnt using 


\begin{tabular}{c|c|l} 
Slot & Requestable & Informable \\
\hline area & yes & $\begin{array}{l}\text { yes. 5 values; north, } \\
\text { south, east, west, centre }\end{array}$ \\
\hline food & yes & yes, 91 possible values \\
\hline name & yes & yes, 113 possible values \\
\hline pricerange & yes & yes, 3 possible values \\
\hline addr & yes & no \\
\hline phone & yes & no \\
\hline postcode & yes & no \\
\hline signature & yes & no
\end{tabular}

Table 1: Ontology used in DSTC2 for restaurant information. Counts do not include the special Dontcare value.

POMDP reinforcement learning

The 2 speech recognisers are:

- ASR-degraded, speech recogniser with artificially degraded statistical acoustic models

- ASR-good, full speech recogniser optimised for the domain

These give two acoustic conditions, the degraded model producing dialogs at higher error rates. The degraded models simulate in-car conditions and are described in Young et al. (2013).

The set of all calls with DM-POMDP, with both speech recognition configurations, constitutes the test set. All calls with the other two dialog managers are used for the training and development set. Specifically, the datasets are arranged as so:

- dstc2_train. Labelled dataset released in October 2013, with 1612 calls from DM-HC and DM-POMDPHC, and both ASR conditions.

- dstc2_dev. Labelled dataset released at the same time as dstc2_train, with 506 calls under the same conditions as dstc2_train. No caller in this set appears in dstc2_train.

- dstc2_test. Set used for evaluation. Released unlabelled at the beginning of the evaluation week. This consists of all 1117 dialogs with DM-POMDP.

Paid Amazon Mechanical Turkers were assigned tasks and asked to call the dialog systems. Callers were asked to find restaurants that matched particular constraints on the slots area, pricerange and food. To elicit more complex dialogs, including changing goals (goals in DSTC1 were always constant), the users were sometimes asked to find more than one restaurant. In cases where a matching restaurant did not exist they were required to seek an alternative, for example finding an Indian instead of an Italian restaurant.

A breakdown of the frequency of goal constraint changes is given in table 2, showing around $40 \%$ of all dialogs involved a change in goal constraint. The distribution of the goal constraints in

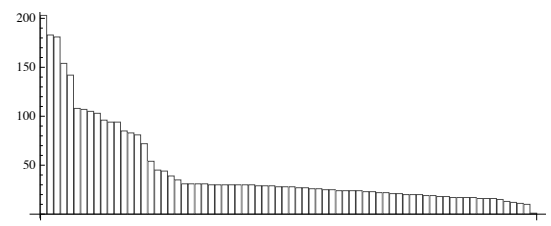

Figure 1: Histogram of values for the food constraint (excluding dontcare) in all data. The most frequent values are Indian, Chinese, Italian and European.

\begin{tabular}{|c|c|c|c|}
\multicolumn{1}{c}{} & \multicolumn{3}{c}{ Dataset } \\
\cline { 2 - 4 } \multicolumn{1}{c|}{} & train & dev & test \\
\hline area & $2.9 \%$ & $1.4 \%$ & $3.8 \%$ \\
\hline food & $37.3 \%$ & $34.0 \%$ & $40.9 \%$ \\
\hline name & $0.0 \%$ & $0.0 \%$ & $0.0 \%$ \\
\hline pricerange & $1.7 \%$ & $1.6 \%$ & $3.1 \%$ \\
\hline any & $40.1 \%$ & $37.0 \%$ & $44.5 \%$ \\
\hline
\end{tabular}

Table 2: Percentage of dialogs which included a change in the goal constraint for each informable (and any slot). Barely any users asked for restaurants by name.

the data was reasonably uniform across the area and pricerange slots, but was skewed for food as shown in figure 1 . The skew arises from the distribution of the restaurants in the system's database; many food types have very few matching venues.

Recently, researchers have started using word confusion networks for spoken language understanding (Henderson et al., 2012; Tür et al., 2013). Unfortunately, word confusion networks were not logged at the time of collecting the dialog data. In order to provide word confusion networks, ASR was run offline in batch mode on each dialog using similar models as the live system. This gives a second set of ASR results, labelled batch, which not only includes ASR $N$-best lists (as in live results), but also word confusion networks.

For each dataset and speech recogniser, table 3 gives the Word Error Rate on the top ASR hypothesis, and F-score for the top SLU hypothesis (calculated as in Henderson et al. (2012)). Note the batch ASR was always less accurate than the live.

\begin{tabular}{|c|c|c|c|c|}
\cline { 3 - 5 } \multicolumn{2}{c|}{} & \multicolumn{2}{c|}{ Live } & Batch \\
\hline Dataset & ASR & WER & F-score & WER \\
\hline \hline \multirow{3}{*}{ train } & degraded & $30.7 \%$ & $72.4 \%$ & $37.7 \%$ \\
& good & $22.4 \%$ & $78.7 \%$ & $25.5 \%$ \\
& all & $26.4 \%$ & $75.7 \%$ & $31.3 \%$ \\
\hline \multirow{3}{*}{ dev } & degraded & $40.4 \%$ & $67.3 \%$ & $47.3 \%$ \\
& good & $25.2 \%$ & $75.2 \%$ & $30.0 \%$ \\
& all & $31.9 \%$ & $71.6 \%$ & $37.6 \%$ \\
\hline \multirow{3}{*}{ test } & degraded & $33.6 \%$ & $70.0 \%$ & $41.1 \%$ \\
& good & $23.5 \%$ & $77.8 \%$ & $27.1 \%$ \\
& all & $28.7 \%$ & $73.8 \%$ & $34.3 \%$ \\
\hline
\end{tabular}

Table 3: Word Error Rate on the top hypothesis, and F-score on top SLU hypothesis. 


\section{Labelling and evaluation}

The output of each tracker is a distribution over dialog states for each turn, as explained in section 2.1. To allow evaluation of the tracker output, the single correct dialog state at each turn is labelled.

Labelling of the dialog state is facilitated by first labelling each user utterance with its semantic representation, in the dialog act format described in Henderson et al. (2013) (some example semantic representations are given in appendix B). The semantic labelling was achieved by first crowdsourcing the transcription of the audio to text. Next a semantic decoder was run over the transcriptions, and the authors corrected the decoder's results by hand. Given the sequence of machine actions and user actions, both represented semantically, the true dialog state is computed deterministically using a simple set of rules.

Recall the dialog state is composed of multiple components; the goal constraint for each slot, the requested slots, and the method. Each of these is evaluated separately, by comparing the tracker output to the correct label. The joint over the goal constraints is evaluated in the same way, where the tracker may either explicitly enumerate and score its joint hypotheses, or let the joint be computed as the product of the distributions over the slots.

A bank of metrics which look at the tracker output and the correct labels are calculated in the evaluation. These metrics are a slightly expanded set of those calculated in DSTC1.

Denote an example probability distribution given by a tracker as $\mathbf{p}$ and the correct label to be $i$, so we have that the probability reported to the correct hypothesis is $\mathbf{p}_{i}$, and $\sum_{j} \mathbf{p}_{j}=1$.

Accuracy measures the fraction of turns where the top hypothesis is correct, i.e. where $i=$ $\arg \max _{j} \mathbf{p}_{j} . \quad \mathbf{A v g P}$, average probability, measures the mean score of the correct hypothesis, $\mathbf{p}_{i}$. This gives some idea of the quality of the score given to the correct hypothesis, ignoring the rest of the distribution. Neglogp is the mean negative logarithm of the score given to the correct hypothesis, $-\log \mathbf{p}_{i}$. Sometimes called the negative log likelihood, this is a standard score in machine learning tasks. MRR is the mean reciprocal rank of the top hypothesis, i.e. $\frac{1}{1+k}$ where $j_{k}=i$ and $\mathbf{p}_{j_{0}} \geq \mathbf{p}_{j_{1}} \geq \ldots$. This metric measures the quality of the ranking, without necessarily treating the scores as probabilities. $\mathbf{L 2}$ measures the square of the $l^{2}$ norm between the distribution and the correct label, indicating quality of the whole reported distribution. It is calculated for one turn as $\left(1-\mathbf{p}_{i}\right)^{2}+\sum_{j \neq i} \mathbf{p}_{j}^{2}$. Two metrics, Update precision and Update accuracy measure the accuracy and precision of updates to the top scoring hypothesis from one turn to the next. For more details, see Higashinaka et al. (2004), which finds these metrics to be highly correlated with dialog success in their data.

Finally there is a set of measures relating to the receiver operating characteristic (ROC) curves, which measure the discrimination of the scores for the highest-ranked hypotheses. Two versions of ROC are computed, V1 and V2. V1 computes correct-accepts (CA), false accepts (FA) and falserejects (FR) as fractions of all utterances. The V2 metrics consider fractions of correctly classified utterances, meaning the values always reach $100 \%$ regardless of the accuracy. V2 metrics measure discrimination independently of the accuracy, and are therefore only comparable between trackers with similar accuracies.

Several metrics are computed from the ROC statistics. ROC V1 EER computes the false acceptance rate at the point where false-accepts are equal to false-rejects. ROC V1 CA05, ROC V1 CA10, ROC V1 CA20 and ROC V2 CA05, ROC V2 CA10, ROC V2 CA20, compute the correct acceptance rates for both versions of ROC at falseacceptance rates $0.05,0.10$, and 0.20 .

Two schedules are used to decide which turns to include when computing each metric. Schedule 1 includes every turn. Schedule 2 only includes a turn if any SLU hypothesis up to and including the turn contains some information about the component of the dialog state in question, or if the correct label is not None. E.g. for a goal constraint, this is whether the slot has appeared with a value in any SLU hypothesis, an affirm/negate act has appeared after a system confirmation of the slot, or the user has in fact informed the slot regardless of the SLU.

The data is labelled using two schemes. The first, scheme A, is considered the standard labelling of the dialog state. Under this scheme, each component of the state is defined as the most recently asserted value given by the user. The None value is used to indicate that a value is yet to be given. Appendix B demonstrates labelling under scheme A.

A second labelling scheme, scheme $\mathbf{B}$, is included in the evaluation, where labels are prop- 
agated backwards through the dialog. This labelling scheme is designed to assess whether a tracker is able to predict a user's intention before it has been stated. Under scheme B, the label at a current turn for a particular component of the dialog state is considered to be the next value which the user settles on, and is reset in the case of goal constraints if the slot value pair is given in a canthelp act by the system (i.e. the system has informed that this constraint is not satisfiable).

\subsection{Featured metrics}

All combinations of metrics, state components, schedules and labelling schemes give rise to 815 total metrics calculated per tracker in evaluation. Although each may have its particular motivation, many of the metrics will be highly correlated. From the results of DSTC1 it was found the metrics could be roughly split into 3 independent groups; one measuring 1-best quality (e.g. Acc), another measuring probability calibration (e.g. L2), and the last measuring discrimination (e.g. ROC metrics) (Williams et al., 2013).

By selecting a representative from each of these groups, the following were chosen as featured metrics:

- Accuracy, schedule 2, scheme A

- L2 norm, schedule 2, scheme A

- ROC V2 CA 5, schedule 2, scheme A

Accuracy is a particularly important measure for dialog management techniques which only consider the top dialog state hypothesis at each turn, while L2 is of more importance when multiple dialog states are considered in action selection. Note that the ROC metric is only comparable among systems operating at similar accuracies, and while L2 should be minimised, Accuracy and the ROC metric should be maximised.

Each of these, calculated for joint goal constraints, search method and combined requested slots, gives 9 metrics altogether which participants were advised to focus on optimizing.

\subsection{Baseline trackers}

Three baseline trackers were entered in the challenge, under the ID 'team0'. Source code for all the baseline systems is available on the DSTC website $^{2}$. The first, 'team0.entry0', follows simple rules commonly used in spoken dialog systems. It gives a single hypothesis for each slot,

\footnotetext{
${ }^{2}$ http://camdial.org/ mh521/dstc/
}

whose value is the top scoring suggestion so far in the dialog. Note that this tracker does not account well for goal constraint changes; the hypothesised value for a slot will only change if a new value occurs with a higher confidence.

The focus baseline, 'team0.entry1', includes a simple model of changing goal constraints. Beliefs are updated for the goal constraint $s=v$, at turn $t, P(s=v)$, using the rule:

$$
P(s=v)_{t}=q_{t} P(s=v)_{t-1}+S L U(s=v)_{t}
$$
where $0 \leq S L U(s=v)_{t} \leq 1$ is the evidence for $s=v$ given by the SLU in turn $t$, and $q_{t}=$ $\sum_{v^{\prime}} S L U\left(s=v^{\prime}\right)_{t} \leq 1$.

Another baseline tracker, based on the tracker presented in Wang and Lemon (2013) is included in the evaluation, labelled 'team0.entry2'. This tracker uses a selection of domain independent rules to update the beliefs, similar to the focus baseline. One rule uses a learnt parameter called the noise adjustment, to adjust the SLU scores. Full details of this and all baseline trackers are provided on the DSTC website.

Finally, an oracle tracker is included under the label 'team0.entry3'. This reports the correct label with score 1 for each component of the dialog state, but only if it has been suggested in the dialog so far by the SLU. This gives an upper-bound for the performance of a tracker which uses only the SLU and its suggested hypotheses.

\section{Results}

Altogether 9 research teams participated in the challenge. Each team could submit a maximum of 5 trackers, and 31 trackers were submitted in total. Teams are identified by anonymous team numbers team1-9, and baseline systems are grouped under team0. Appendix A gives the results on the featured metrics for each entry submitted to the challenge. The full results, including tracker output, details of each tracker and scripts to run the evaluation are available on the DSTC2 website.

The table in appendix A specifies which of the inputs available were used for each tracker- from live ASR, live SLU and batch ASR. This facilitates comparisons between systems which used the same information.

A variety of techniques were used in the submitted trackers. Some participants provided short synopses, which are available in the download from the DSTC2 website. Full details on the trackers themselves are published at SIGdial 2014. 
For the "requested slot" task, some trackers outperformed the oracle tracker. This was possible because trackers could guess a slot was requested using dialog context, even if there was no mention of it in the SLU output.

Participants were asked to report the results of their trackers on the dstcs2_dev development set. Figure 2 gives some insight into how well performance on the development set predicted performance on the test set. Metrics are reported as percentage improvement relative to the focus baseline to normalise for the difficulty of the datasets; in general trackers achieved higher accuracies on the test set than on development. Figure 2 shows that the development set provided reasonable predictions, though in all cases improvement relative to the baseline was overestimated, sometimes drastically. This suggests that approaches to tracking have trouble with generalisation, underperforming in the mismatched conditions of the test set which used an unseen dialog manager.

Joint Goal Constraint Accuracy

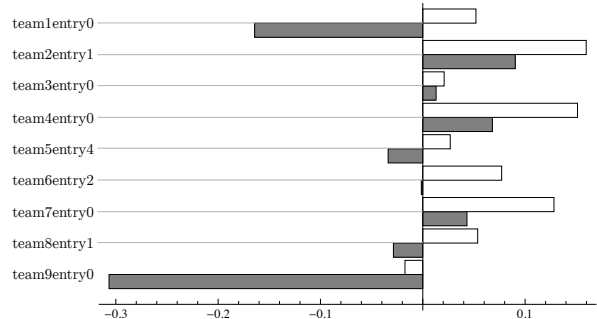

Joint Goal Constraint L2

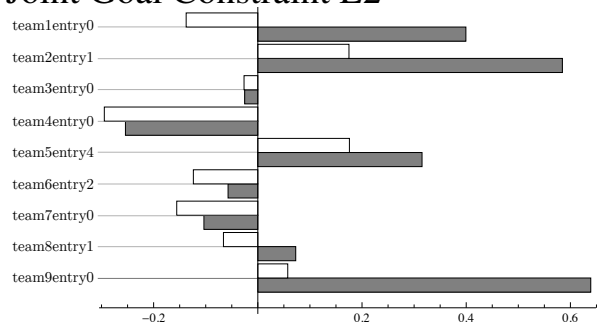

Figure 2: Performance relative to the focus baseline (percentage increase) for dev set (white) and test set (grey). Top entry for each team chosen based on joint goal constraint accuracy. A lower L2 score is better.

Recall from section 2, trackers could output joint distributions for goal constraints, or simply output one distribution for each slot and allow the joint to be calculated as the product. Two teams, team 2 and team8, opted to output a joint distribution for some of their entries. Figure 3 compares performance on the test set for these trackers between the joint distributions they reported, and the joint calculated as the product. The entries from team 2 were able to show an increase in the accu- racy of the top joint goal constraint hypotheses, but seemingly at a cost in terms of the L2 score. Conversely the entries from team8, though operating at lower performance than the focus baseline, were able to show an improvement in L2 at a slight loss in accuracy. These results suggest that a tracking method is yet to be proposed which can, at least on this data, improve both accuracy and the L2 score of tracker output by reporting joint predictions of goal constraints.
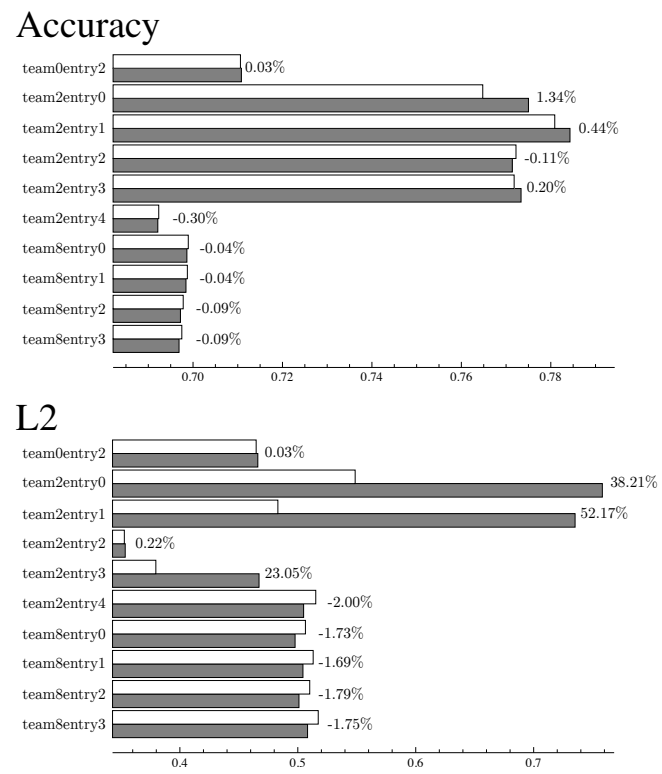

Figure 3: Influence of reporting a full joint distribution. White bar shows test set performance computing the goal constraints as a product of independent marginals; dark bar is performance with a full joint distribution. All entries which reported a full joint are shown. A lower L2 score is better.

It is of interest to investigate the correlation between accuracy and L2. Figure 4 plots these metrics for each tracker on joint goal constraints. We see that in general a lower L2 score correlates with a higher accuracy, but there are examples of high accuracy trackers which do poorly in terms of L2. This further justifies the reporting of these as two separate featured metrics.

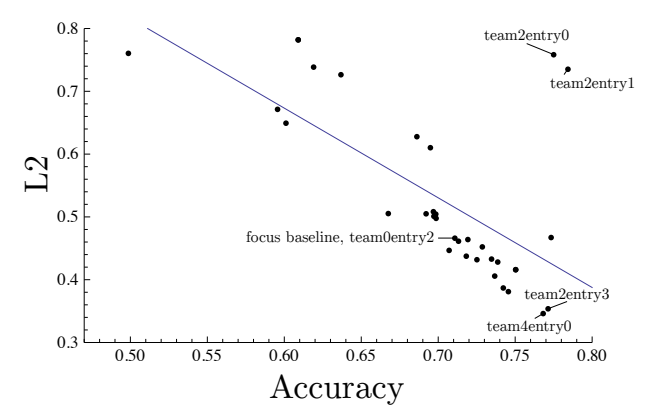

Figure 4: Scatterplot of joint goal constraint accuracy and joint goal constraint L2 for each entry. Plotted line is leastsquares linear regression, L2 $=1.53-1.43$ Accuracy 


\begin{tabular}{c||cc|cc|cc}
\multicolumn{1}{c||}{ Tracker } & \multicolumn{2}{c}{ Joint goal } & \multicolumn{2}{c}{ Method } & \multicolumn{2}{c}{ Requested } \\
& Acc. & L2 & Acc. & L2 & Acc. & L2 \\
\hline \hline Single best entry & 0.784 & 0.346 & $\mathbf{0 . 9 5 0}$ & $\mathbf{0 . 0 8 2}$ & 0.978 & 0.035 \\
\hline Score averaging: top 2 entries & 0.787 & $0.364-$ & $0.945-$ & 0.083 & 0.976 & $0.039-$ \\
Score averaging: top 5 entries & 0.777 & 0.347 & 0.945 & $0.089-$ & 0.976 & 0.038 \\
Score averaging: top 10 entries & $0.760-$ & $0.364-$ & $0.934-$ & $0.108-$ & $0.967-$ & $0.056-$ \\
Score averaging: all entries & $0.765-$ & $0.362-$ & $0.934-$ & $0.103-$ & $0.971-$ & $0.052-$ \\
\hline Stacking: top 2 entries & 0.789 & $0.322+$ & 0.949 & $0.085-$ & 0.977 & $0.040-$ \\
Stacking: top 5 entries & $0.795+$ & $0.315+$ & 0.949 & 0.084 & 0.978 & 0.037 \\
Stacking: top 10 entries & $0.796+$ & $0.312+$ & 0.949 & 0.083 & 0.979 & 0.035 \\
Stacking: all entries & $\mathbf{0 . 7 9 8 +}$ & $\mathbf{0 . 3 0 8}+$ & $\mathbf{0 . 9 5 0}$ & 0.083 & $\mathbf{0 . 9 8 0}$ & $\mathbf{0 . 0 3 4}$
\end{tabular}

Table 4: Accuracy and L2 for Joint goal constraint, Method, and Requested slots for the single best tracker (by accuracy) in DSTC2, and various ensemble methods. "Top N entries" means the $\mathrm{N}$ entries with highest accuracies from distinct teams, where the baselines are included as a team. + /- indicates statistically significantly better/worse than the single best entry $(p<0.01)$, computed with McNemar's test for accuracy and the paired t-test for L2, both with Bonferroni correction for repeated tests.

\section{Ensemble learning}

The dialog state tracking challenge provides an opportunity to study ensemble learning - i.e. synthesizing the output of many trackers to improve performance beyond any single tracker. Here we consider two forms of ensemble learning: score averaging and stacking.

In score averaging, the final score of a class is computed as the mean of the scores output by all trackers for that class. One of score averaging's strengths is that it requires no additional training data beyond that used to train the constituent trackers. If each tracker's output is correct more than half the time, and if the errors made by trackers are not correlated, then score averaging is guaranteed to improve performance (since the majority vote will be correct in the limit). In (Lee and Eskenazi, 2013), score averaging (there called "system combination") has been applied to combine the output of four dialog state trackers. To help decorrelate errors, constituent trackers were trained on different subsets of data, and used different machine learning methods. The relative error rate reduction was $5.1 \%$ on the test set.

The second approach to ensemble learning is stacking (Wolpert, 1992). In stacking, the scores output by the constituent classifiers are fed to a new classifier that makes a final prediction. In other words, the output of each constituent classifier is viewed as a feature, and the new final classifier can learn the correlations and error patterns of each. For this reason, stacking often outperforms score averaging, particularly when errors are correlated. However, stacking requires a validation set for training the final classifier. In DSTC2, we only have access to trackers' output on the test set. Therefore, to estimate the performance of stacking, we perform cross-validation on the test set: the test set is divided into two folds. First, fold 1 is used for training the final classifier, and fold 2 is used for testing. Then the process is reversed. The two test outputs are then concatenated. Note that models are never trained and tested on the same data. A maximum entropy model (maxent) is used (details in (Metallinou et al., 2013)), which is common practice for stacking classifiers. In addition, maxent was found to yield best performance in DSTC1 (Lee and Eskenazi, 2013).

Table 4 reports accuracy and L2 for goal constraints, search method, and requested slots. For each ensemble method and each quantity (column) the table gives results for combining the top trackers from 2 or 5 distinct teams, for combining the top tracker from each team, and combining all trackers (including the baselines as a team). For example, the joint goal constraint ensemble with the top 2 entries was built from team2.entry $1 \&$ team4.entry0, and the method ensemble with the top 2 entries from team2.entry $4 \&$ team4.entry0.

Table 4 shows two interesting trends. The first is that score averaging does not improve performance, and performance declines as more trackers are combined, yielding a statistically significant decrease across all metrics. This suggests that the errors of the different trackers are correlated, which is unsurprising since they were trained on the same data. On the other hand, stacking yields a statistically significant improvement in accuracy for goal constraints, and doesn't degrade accuracy for the search method and requested slots. For stacking, the trend is that adding more trackers increases performance - for example, combining the best tracker from every team improves goal constraint accuracy from $78.4 \%$ to $79.8 \%$.

For completeness, we note that the additional data could alternatively be used to improve the accuracy of a constituent classifier; given the constraints of the challenge, we can't assess the mag- 
nitude of that improvement, so it is an open question whether stacking is the best use of additional data. Also, the training and test conditions of the final stacking classifier are not mis-matched, whereas in practice they would be. Nonetheless, this result does suggest that, if additional data is available, stacking can be used to successfully combine multiple trackers and achieve performance better than the single best tracker.

\section{Conclusions}

DSTC2 continues the tradition of DSTC1 by providing a common testbed for dialog state tracking, introducing some additional features relevant to the research community- specifically a new domain, changing user goals and a richer dialog state. The data, evaluation scripts, and baseline trackers will remain available and open to the research community online.

Results from the previous challenge motivated the selection of a few metrics as featured metrics, which facilitate comparisons between trackers. Analysis of the performance on the matched development set and the mismatched test set suggests that there still appears to be limitations on generalisation, as found in DSTC1. The results also suggest there are limitations in exploiting correlations between slots, with few teams exploiting joint distributions and the effects of doing so being mixed. Investigating ensemble learning demonstrates the effectiveness of combining tracker outputs. Ensemble learning exploits the strengths of individual trackers to provide better quality output than any constituent tracker in the group.

A follow up challenge, DSTC3, will present the problem of adapting to a new domain with very few example dialogs. Future work should also verify that improvements in dialog state tracking translate to improvements in end-to-end dialog system performance. In this challenge, paid subjects were used as users with real information needs were not available. However, differences between these two user groups have been shown (Raux et al., 2005), so future studies should also test on real users.

\section{Acknowledgements}

The authors thank the advisory committee for their valuable input: Paul Crook, Maxine Eskenazi, Milica Gašić, Helen Hastie, Kee-Eung Kim, Sungjin Lee, Oliver Lemon, Olivier Pietquin, Joelle Pineau, Deepak Ramachandran, Brian
Strope and Steve Young. The authors also thank Zhuoran Wang for providing a baseline tracker, and DJ Kim, Sungjin Lee \& David Traum for comments on evaluation metrics. Finally, thanks to SIGdial for their endorsement, and to the participants for making the challenge a success.

\section{References}

Matthew Henderson, Milica Gašić, Blaise Thomson, Pirros Tsiakoulis, Kai Yu, and Steve Young. 2012. Discriminative Spoken Language Understanding Using Word Confusion Networks. In Spoken Language Technology Workshop, 2012. IEEE.

Matthew Henderson, Blaise Thomson, and Jason Williams. 2013. Dialog State Tracking Challenge 2 \& 3 Handbook. camdial.org/ mh521/dstc/.

Ryuichiro Higashinaka, Noboru Miyazaki, Mikio Nakano, and Kiyoaki Aikawa. 2004. Evaluating discourse understanding in spoken dialogue systems. ACM Trans. Speech Lang. Process., November.

Sungjin Lee and Maxine Eskenazi. 2013. Recipe for building robust spoken dialog state trackers: Dialog state tracking challenge system description. In Proceedings of the SIGDIAL 2013 Conference.

Angeliki Metallinou, Dan Bohus, and Jason D. Williams. 2013. Discriminative state tracking for spoken dialog systems. In Proc Association for Computational Linguistics, Sofia.

Antoine Raux, Brian Langner, Dan Bohus, Alan W Black, and Maxine Eskenazi. 2005. Let's go public! Taking a spoken dialog system to the real world.

Gökhan Tür, Anoop Deoras, and Dilek Hakkani-Tür. 2013. Semantic parsing using word confusion networks with conditional random fields. In INTERSPEECH.

Zhuoran Wang and Oliver Lemon. 2013. A simple and generic belief tracking mechanism for the dialog state tracking challenge: On the believability of observed information. In Proceedings of the SIGDIAL 2013 Conference.

Jason Williams, Antoine Raux, Deepak Ramachadran, and Alan Black. 2013. The Dialog State Tracking Challenge. In Proceedings of the SIGDIAL 2013 Conference, Metz, France, August.

David H. Wolpert. 1992. Stacked generalization. Neural Networks, 5:241-259.

Steve Young, Catherine Breslin, Milica Gašić, Matthew Henderson, Dongho Kim, Martin Szummer, Blaise Thomson, Pirros Tsiakoulis, and Eli Tzirkel Hancock. 2013. Evaluation of Statistical POMDP-based Dialogue Systems in Noisy Environment. In Proceedings of IWSDS, Napa, USA, January. 


\section{Appendix A: Featured results of evaluation}

\begin{tabular}{|c|c|c|c|c|c|c|c|c|c|c|c|c|c|}
\hline \multirow[b]{2}{*}{ team } & \multirow[b]{2}{*}{ entry } & \multicolumn{3}{|c|}{ Tracker Inputs } & \multicolumn{3}{|c|}{ Joint Goal Constraints } & \multicolumn{3}{|c|}{ Search Method } & \multicolumn{3}{|c|}{ Requested Slots } \\
\hline & & $\begin{array}{l}\text { Live } \\
\text { ASR }\end{array}$ & $\begin{array}{l}\text { Live } \\
\text { SLU }\end{array}$ & $\begin{array}{l}\text { Batch } \\
\text { ASR }\end{array}$ & Acc & L2 & ROC & Acc & L2 & ROC & Acc & L2 & ROC \\
\hline \multirow[t]{4}{*}{$0^{*}$} & 0 & & $\checkmark$ & & 0.619 & 0.738 & 0.000 & 0.879 & 0.209 & 0.000 & 0.884 & 0.196 & 0.000 \\
\hline & 1 & & $\checkmark$ & & 0.719 & 0.464 & 0.000 & 0.867 & 0.210 & 0.349 & 0.879 & 0.206 & 0.000 \\
\hline & 2 & & $\checkmark$ & & 0.711 & 0.466 & 0.000 & 0.897 & 0.158 & 0.000 & 0.884 & 0.201 & 0.000 \\
\hline & 3 & & $\checkmark^{\dagger}$ & & 0.850 & 0.300 & 0.000 & 0.986 & 0.028 & 0.000 & 0.957 & 0.086 & 0.000 \\
\hline \multirow[t]{2}{*}{1} & 0 & & $\checkmark$ & & 0.601 & 0.649 & 0.064 & 0.904 & 0.155 & 0.187 & 0.960 & 0.073 & 0.000 \\
\hline & 1 & & $\checkmark$ & & 0.596 & 0.671 & 0.036 & 0.877 & 0.204 & 0.397 & 0.957 & 0.081 & 0.000 \\
\hline \multirow[t]{5}{*}{2} & 0 & $\checkmark$ & $\checkmark$ & & 0.775 & 0.758 & 0.063 & 0.944 & 0.092 & 0.306 & 0.954 & 0.073 & 0.383 \\
\hline & 1 & $\checkmark$ & $\checkmark$ & $\checkmark$ & 0.784 & 0.735 & 0.065 & 0.947 & 0.087 & 0.355 & 0.957 & 0.068 & 0.446 \\
\hline & 2 & $\checkmark$ & & & 0.668 & 0.505 & 0.249 & 0.944 & 0.095 & 0.499 & 0.972 & 0.043 & 0.300 \\
\hline & 3 & $\checkmark$ & $\checkmark$ & $\checkmark$ & 0.771 & 0.354 & 0.313 & 0.947 & 0.093 & 0.294 & 0.941 & 0.090 & 0.262 \\
\hline & 4 & $\checkmark$ & $\checkmark$ & $\checkmark$ & 0.773 & 0.467 & 0.140 & 0.950 & 0.082 & 0.351 & 0.968 & 0.050 & 0.497 \\
\hline 3 & 0 & & $\checkmark$ & & 0.729 & 0.452 & 0.000 & 0.878 & 0.210 & 0.000 & 0.889 & 0.188 & 0.000 \\
\hline \multirow[t]{4}{*}{4} & 0 & $\checkmark$ & & & 0.768 & 0.346 & 0.365 & 0.940 & 0.095 & 0.452 & 0.978 & 0.035 & 0.525 \\
\hline & 1 & $\checkmark$ & & & 0.746 & 0.381 & 0.383 & 0.939 & 0.097 & 0.423 & 0.977 & 0.038 & 0.490 \\
\hline & 2 & & $\checkmark$ & & 0.742 & 0.387 & 0.345 & 0.922 & 0.124 & 0.447 & 0.957 & 0.069 & 0.340 \\
\hline & 3 & & $\checkmark$ & & 0.737 & 0.406 & 0.321 & 0.922 & 0.125 & 0.406 & 0.957 & 0.073 & 0.385 \\
\hline \multirow[t]{5}{*}{5} & 0 & $\checkmark$ & $\checkmark$ & & 0.686 & 0.628 & 0.000 & 0.889 & 0.221 & 0.000 & 0.868 & 0.264 & 0.000 \\
\hline & 1 & $\checkmark$ & $\checkmark$ & & 0.609 & 0.782 & 0.000 & 0.927 & 0.147 & 0.000 & 0.974 & 0.053 & 0.000 \\
\hline & 2 & $\checkmark$ & $\checkmark$ & & 0.637 & 0.726 & 0.000 & 0.927 & 0.147 & 0.000 & 0.974 & 0.053 & 0.000 \\
\hline & 3 & $\checkmark$ & $\checkmark$ & & 0.609 & 0.782 & 0.000 & 0.927 & 0.147 & 0.000 & 0.974 & 0.053 & 0.000 \\
\hline & 4 & $\checkmark$ & $\checkmark$ & & 0.695 & 0.610 & 0.000 & 0.927 & 0.147 & 0.000 & 0.974 & 0.053 & 0.000 \\
\hline \multirow[t]{3}{*}{6} & 0 & & $\checkmark$ & & 0.713 & 0.461 & 0.100 & 0.865 & 0.228 & 0.199 & 0.932 & 0.118 & 0.057 \\
\hline & 1 & & $\checkmark$ & & 0.707 & 0.447 & 0.223 & 0.871 & 0.211 & 0.290 & 0.947 & 0.093 & 0.218 \\
\hline & 2 & & $\checkmark$ & & 0.718 & 0.437 & 0.207 & 0.871 & 0.210 & 0.287 & 0.951 & 0.085 & 0.225 \\
\hline \multirow[t]{5}{*}{7} & 0 & $\checkmark$ & & & 0.750 & 0.416 & 0.081 & 0.936 & 0.105 & 0.237 & 0.970 & 0.056 & 0.000 \\
\hline & 1 & $\checkmark$ & & & 0.739 & 0.428 & 0.159 & 0.921 & 0.161 & 0.554 & 0.970 & 0.056 & 0.000 \\
\hline & 2 & $\checkmark$ & & & 0.750 & 0.416 & 0.081 & 0.929 & 0.117 & 0.379 & 0.971 & 0.054 & 0.000 \\
\hline & 3 & $\checkmark$ & & & 0.725 & 0.432 & 0.105 & 0.936 & 0.105 & 0.237 & 0.972 & 0.047 & 0.000 \\
\hline & 4 & & $\checkmark$ & & 0.735 & 0.433 & 0.086 & 0.910 & 0.140 & 0.280 & 0.946 & 0.089 & 0.190 \\
\hline \multirow[t]{5}{*}{8} & 0 & & $\checkmark$ & & 0.692 & 0.505 & 0.071 & 0.899 & 0.153 & 0.000 & 0.935 & 0.106 & 0.000 \\
\hline & 1 & & $\checkmark$ & & 0.699 & 0.498 & 0.067 & 0.899 & 0.153 & 0.000 & 0.939 & 0.101 & 0.000 \\
\hline & 2 & & $\checkmark$ & & 0.698 & 0.504 & 0.067 & 0.899 & 0.153 & 0.000 & 0.939 & 0.101 & 0.000 \\
\hline & 3 & & $\checkmark$ & & 0.697 & 0.501 & 0.068 & 0.899 & 0.153 & 0.000 & 0.939 & 0.101 & 0.000 \\
\hline & 4 & & $\checkmark$ & & 0.697 & 0.508 & 0.068 & 0.899 & 0.153 & 0.000 & 0.939 & 0.101 & 0.000 \\
\hline 9 & 0 & & $\checkmark$ & & 0.499 & 0.760 & 0.000 & 0.857 & 0.229 & 0.000 & 0.905 & 0.149 & 0.000 \\
\hline
\end{tabular}

$*$ The entries under team 0 are the baseline systems mentioned in section $3.2 .{ }^{\dagger}$ team0.entry 3 is the oracle tracker, which uses the labels on the test set and limits itself to hypotheses suggested by the live SLU.

The top score in each column is indicated by bold-type. The ROC metric is only comparable for trackers operating at a similar accuracy, and so the highest values are not indicated. 
Appendix B: Sample dialog, labels, and tracker output

\begin{tabular}{|c|c|c|c|c|}
\hline Actual input and output & SLU hypotheses and scores & Labels & Example tracker output & Correct? \\
\hline \multirow{3}{*}{$\begin{array}{l}\text { S: Which part of town? } \\
\text { request(area) }\end{array}$} & 0.2 inform(food=north_african) & area=north & 0.2 food=north_african & $x$ \\
\hline & 0.1 inform(area=north) & & 0.1 area $=$ north & \\
\hline & & & 0.7() & $x$ \\
\hline \multirow[t]{2}{*}{$\begin{array}{l}\text { U: The north uh area } \\
\text { inform(area=north) }\end{array}$} & & method=byconstraints & $\begin{array}{l}0.9 \text { byconstraints } \\
0.1 \text { none }\end{array}$ & \\
\hline & & requested=() & $\begin{array}{l}0.0 \text { phone } \\
0.0 \text { address }\end{array}$ & \\
\hline $\begin{array}{l}\text { S: Which part of town? } \\
\text { request(area) }\end{array}$ & $\begin{array}{l}0.8 \text { inform(area=north), } \\
\text { inform(pricerange=cheap) }\end{array}$ & $\begin{array}{l}\text { area=north } \\
\text { pricerange=cheap }\end{array}$ & $\begin{array}{l}0.7 \text { area=north } \\
\text { pricerange=cheap }\end{array}$ & \\
\hline \multirow{4}{*}{$\begin{array}{l}\text { U: A cheap place in } \\
\text { the north } \\
\text { inform (area=north, } \\
\text { pricerange=cheap) }\end{array}$} & 0.1 inform(area=north) & & $\begin{array}{l}0.1 \text { area=north } \\
\text { food=north_african }\end{array}$ & $\gamma$ \\
\hline & & & 0.2() & $x$ \\
\hline & & method=byconstraints & $\begin{array}{l}0.9 \text { byconstraints } \\
0.1 \text { none }\end{array}$ & \\
\hline & & requested $=()$ & $\begin{array}{l}0.0 \text { phone } \\
0.0 \text { address }\end{array}$ & \\
\hline \multirow{5}{*}{$\begin{array}{l}\text { S: Clown café is a cheap } \\
\text { restaurant in the } \\
\text { north part of town. } \\
\text { U: Do you have any } \\
\text { others like that, } \\
\text { maybe in the south } \\
\text { part of town? } \\
\text { reqalts(area=south) }\end{array}$} & 0.7 reqalts(area $=$ south) & $\begin{array}{l}\text { area=south } \\
\text { pricerange=cheap }\end{array}$ & $\begin{array}{l}0.8 \text { area=south } \\
\text { pricerange=cheap }\end{array}$ & \\
\hline & 0.2 reqmore() & & $\begin{array}{l}0.1 \text { area=north } \\
\text { pricerange=cheap }\end{array}$ & \\
\hline & & & 0.1() & $x$ \\
\hline & & method=byalternatives & $\begin{array}{l}0.6 \text { byalternatives } \\
0.2 \text { byconstraints }\end{array}$ & \\
\hline & & requested $=()$ & $\begin{array}{l}0.0 \text { phone } \\
0.0 \text { address }\end{array}$ & $\checkmark$ \\
\hline \multirow{5}{*}{$\begin{array}{l}\text { S: Galleria is a cheap } \\
\text { restaurant in the } \\
\text { south. } \\
\text { U: What is their phone } \\
\text { number and } \\
\text { address? } \\
\text { request(phone), } \\
\text { request(address) }\end{array}$} & 0.6 request(phone) & $\begin{array}{l}\text { area }=\text { south } \\
\text { pricerange=cheap }\end{array}$ & $\begin{array}{l}0.9 \text { area=south } \\
\text { pricerange=cheap }\end{array}$ & \\
\hline & $\begin{array}{l}0.2 \text { request(phone), } \\
\text { request(address) }\end{array}$ & & $\begin{array}{l}0.1 \text { area=north } \\
\text { pricerange=cheap }\end{array}$ & $x$ \\
\hline & 0.1 request(address) & & 0.0() & $x$ \\
\hline & & method=byalternatives & $\begin{array}{l}0.5 \text { byconstraints } \\
0.4 \text { byalternatives }\end{array}$ & $\mathbf{X}$ \\
\hline & & $\begin{aligned} \text { requested }= & \text { (phone, } \\
& \text { address) }\end{aligned}$ & $\begin{array}{l}0.8 \text { phone } \\
0.3 \text { address }\end{array}$ & $\sqrt{x}$ \\
\hline
\end{tabular}

Example dialog illustrating DSTC2 data, labels, and evaluation procedure. The left column shows the actual system output and user input. The second column shows two SLU N-Best hypothesis and their scores. In practice, up to 10 SLU N-Best hypotheses are output. In the right 3 columns, the three shaded regions correspond to the three components of the dialog state output by a tracker at each turn. The blue region corresponds to the user's joint goal constraint; the red region to the user's search method; and the yellow region to the slots requested by the user. For space, only 2 of the 5 methods and 2 of the 8 requestable slots are shown. The third column shows the label (correct output) for each component. The fourth column shows example tracker output for each of these three quantities, and the fifth column indicates correctness. A goal constraint is correct if it exactly matches the label. Therefore, 0 or 1 of the output goal constraints is correct, and all the others are incorrect. Accuracy is determined by the correctness of the goal constraint with the highest tracker score. For search method, exactly one method is correct at each turn, so correctness is determined by comparing the maximum scoring method to the label. For requested slots, each slot can be requested (or not) in the same turn, so each requestable slot is separately marked as correct or incorrect. The quantity requested.all averages the correctness of all requested slots. 